Reprod. Nutr. Dévelop., 1980, 20 (4 B), 1187-1192.

\title{
Caractéristiques de la compartimentation fonctionnelle de l'intestin grêle. Etude de l'étage duodénal chez le Rat
}

par M. C. LEMAIRE, M. LEGOUX, Odette MORELLE, J. THOUVENOT

Laboratoire de Physiologie, UER Médecine de l'Université de Tours, B. P. 3223, 37032 Tours Cedex, France

Summary. Functional partition of the small intestine : duodenal level in the rat.

A radiological study using the conscious rat $(n=23)$ showed the different putative compartments at the duodenal level.

We report here the in vitro pressure-volume-time of two compartments.

The first one extended 0 to $1.7 \mathrm{~cm} \pm 0.3$ from the pylorus, while the second one included the junction with the common biliopancreatic duct.

The significant results showed that the first compartment had relative stretch with predominant pendulum motility ; the threshold stretch for the onset of this motility was 58 p. 100 of the mean maximum diameter. It thus appeared to have a capacitive function, whereas the second compartment acted as a propulsion system in which the threshold stretch for peristalsis was 29 p. 100 of the mean maximum diameter.

\section{Introduction.}

Le présent travail consiste à préciser, in vitro, les caractérisłiques pression-volumetemps des deux premiers compartiments duodénaux définis lors du transit baryté, in vivo, chez le Rat éveillé. L'étude du transit baryté chez l'animal a repris une nouvelle extension grâce aux méthodes d'analyse quantitative d'images qui permettent de préciser les temps de réplétion et d'évacuation des divers étages. Ainsi, on a pu montrer que l'évacuation gastrique d'une suspension barytée avec du saccharose est significativement plus lente que pour le glucose ou le lactose, à concentrations isoénergétiques et dans les mêmes conditions (Morelle et al., 1979).

\section{Matériel et méthodes.}

Nous utilisons pour l'étape radiologique, des rats mâles Wistar $(n=23)$. Ceuxci reçoivent par intubation $2 \mathrm{ml}$ d'une suspension barytée *, puis sont placés dans une

* Solution $\left(0,22 \mathrm{M} \cdot \mathrm{I}^{-1}\right)$; Dose $\cdot\left(22 \cdot 10^{-4} \mathrm{M} \cdot \mathrm{kg}^{-1}\right)$. 
enceinte de contention adaptée à la prise de clichés radiologiques (Morelle, 1978). Pour l'étude du temps radiologique gastroduodénal, les clichés s'échelonnent de la première minute à la deuxième heure après intubation selon les temps suivants : $1,2,3,4,5,10,15,30,45,60,90$ et $120 \mathrm{~min}$.

Ełude in vivo. - Les images duodénales sont digitalisées au moyen d'un capteur optique (Rivaillier et al., 1978). On repère les minimum du contour duodénal $\sigma_{1} \sigma_{2}$ qui indiquent les limites des compartiments. Pour caractériser ces compartiments, on compte les niveaux de gris correspondant à la réplétion baryłée.

Ełude in vitro. - Pour cette éłude $(n=20)$, on prélève le fragment duodénal correspondant à la délimitation définie radiologiquement. Après laparotomie médiane et anesthésie au pentobarbital sodique, le segment choisi est prélevé rapidement et monté sur deux canules orale ef aborale qui peuvent être adaptées au dispositif myographique, le tout mis en place dans une cuve thermostatée remplie de solution saline* à $37^{\circ} \mathrm{C}$ avec barbotage d'air (fig. 1).

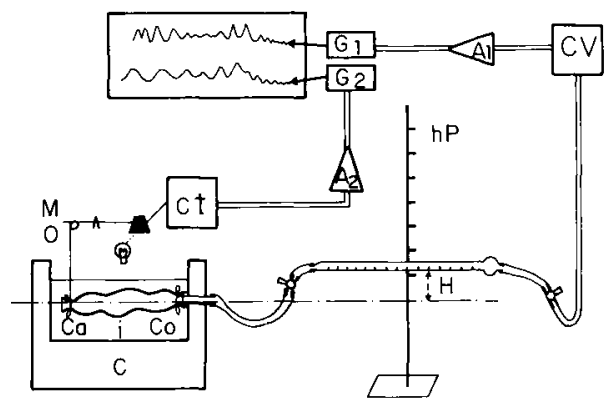

FIG. 1. - Dispositif bioméconique in vitro. Dans une cuve thermostatique $C$ solidaire d'un circuit de chauffage est monté le segment intestinal i dont la lumière est en relation avec la canule orale Co et aborale $\mathrm{Ca}$. Co est reliée au système de distension à pression imposée par la hauteur $\mathrm{H}$ de la pipette horizontale. Les variations de volume sont mesurées au niveau du capteur Cr. La canule aborale Ca fermée est reliée au myographe isotonique $M$, mobile autour de 0 et dont les déplacements sont transmis par le capteur photoélectrique $C$. Les amplificateurs $A_{1}$ et $A_{2}$ alimentent les scripteurs $G_{1}$ et $G_{2}$.

La canule orale est en relation avec un circuit solidaire d'une pipette horizontale mobile sur une glissière verticale permettant d'imposer une pression connue et constante au compartiment intestinal étudié. La canule aborale obturée est solidaire du levier myographique qui enregistre les variations de longueur du fragment intestinal sous tension constante $\left(4,9 \cdot 10^{-3} \mathrm{~N}\right)$.

Pour les enregistrements, on utilise les capteurs à transmission photoélectrique : - CV, en relation avec l'autre extrémité de la pipette, est constitué d'une capsule à membrane à grande compliance $\left(2,5 \cdot 10^{-3} \mathrm{ml} \mathrm{Pa}^{-1}\right)$ dans la zone de linéarité.

* Composition saline, type Tyrode (en $\mathrm{mM}$ ) :

$\mathrm{NaCl} \quad 136,75 \quad \mathrm{CaCl}_{2} \quad 1,80$

$\mathrm{KCl} \quad 0,67 \quad \mathrm{MgCl}_{2} 1,05$ Bicarbonate 16,13 eau Q. S. p. 1000. 
- CT solidaire d'un cache obturant partiellement un système à transmission photoélectrique. Les enregistrements sont réalisés au moyen d'un polygraphe (Compagnie des Compteurs, Division Schlumberger) sur microfilm avec microscripteurs (10 mA) ef amplificateurs, ou d'un enregistreur potentiométrique Tacussel (Type EPL1).

\section{Résultats.}

1. - Ełude radiologique (fig. 2). - Au cours des radiographies successives lors du transit baryté (intervalle $1 \mathrm{~min}$ ). on met en évidence des zones de rétrécissement que

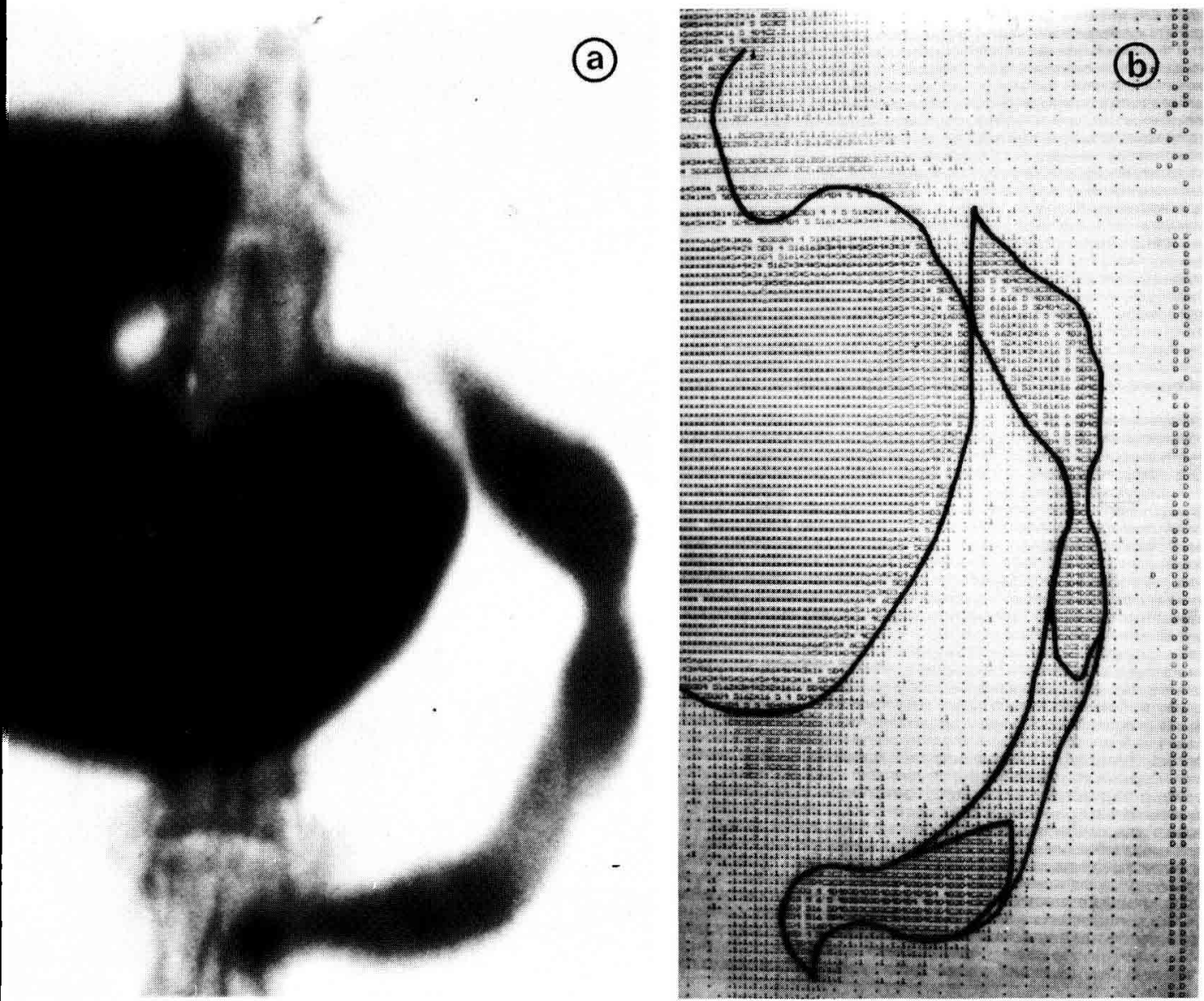

FIG. 2. - a) Rodiographie duodénale au cours d'un transit baryté. Cliché 1 min après intubation. b) Digitalisation de l'image précédente en 12 niveaux de gris permettant de coractériser les compartiments. L'absorption barytée correspond aux niveaux 5-12.

l'on retrouve pratiquement sur tous les premiers clichés (phase duodénale) : on délimite ainsi trois compartiments duodénaux (tabl. 1). Ces compartiments ont les caractéristiques suivantes : 
TABLEAU 1

\begin{tabular}{lcccc}
\hline & $\begin{array}{c}\text { Origine à partir } \\
\text { du pylore en } \mathrm{cm}\end{array}$ & $\begin{array}{c}\text { Longueur } \\
\mathrm{cm}\end{array}$ & $\begin{array}{c}\text { Surface de } \\
\text { projections en } \mathrm{cm}^{2}\end{array}$ \\
\hline $\mathrm{CD}_{1}$ & 0 & $1,7 \pm 0,3$ & $0,76 \pm 0,23$ & $\mathrm{n}=8$ \\
\hline $\mathrm{CD}_{2}$ & $1,7 \pm 0,3$ & $2,5 \pm 0,4$ & $0,52 \pm 0,17$ & $\mathrm{n}=9$ \\
\hline $\mathrm{CD}_{3}$ & $4,2 \pm 0,7$ & $2,8 \pm 0,4$ & $0,75 \pm 0,26 \quad \mathrm{n}=6$ \\
\hline
\end{tabular}

2. - Ełude biomécanique in vitro (fig. 3). - Les expériences n'ont porté ici que sur les compartiments $C D_{1}$ et $C D_{2}$ : on établit ainsi le diagramme représentant le volume intraluminal moyen en fonction des pressions intraluminales imposées (en fonction du temps selon le protocole).
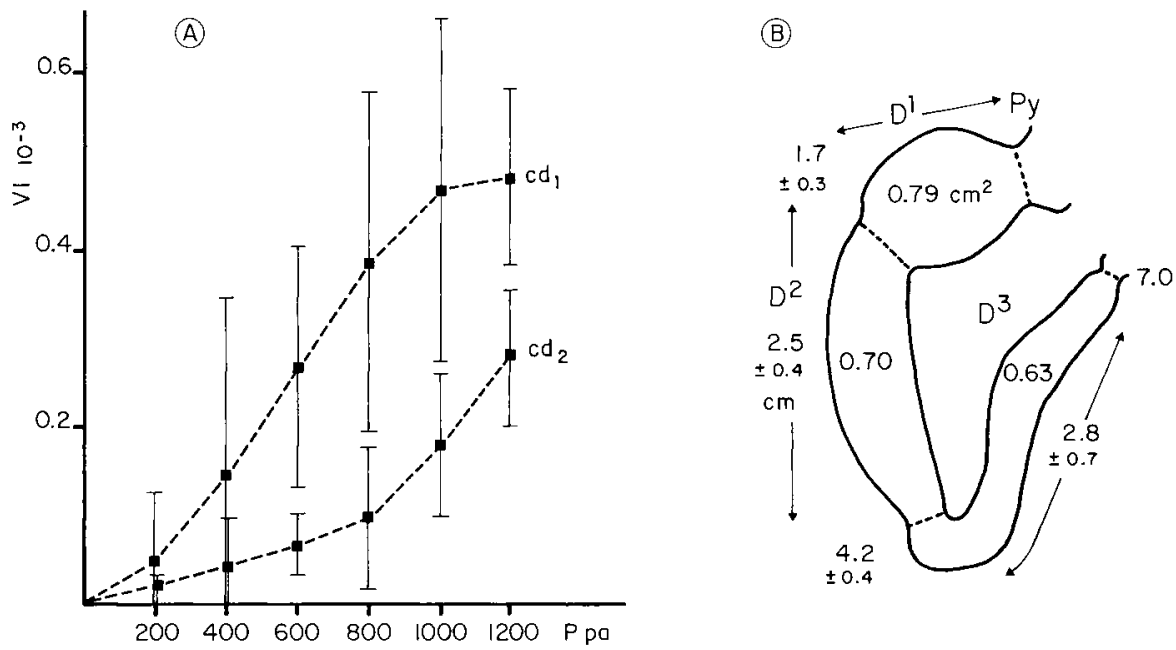

FIG. 3. - A) Diagramme représentant pour les compartiments $C D_{1}$ et $C D_{2}$ le volume intraluminal moyen (ordonnées en $\mathrm{I}_{10^{-3}}$ ) en fonction de la pression imposée (abscisses en Pascals). Les écarts types $\sigma$ sont indiqués par des traits verticaux. B) Schématisation de la défermination radiologique des segments duodénaux. Les zones de rétrécissement $\delta_{1}, \delta_{2}, \delta_{3}$ délimitent les compartiments duodénaux $\mathrm{CD}_{1}$, $C D_{2}, C D_{3}$ dans le cadre d'une symbolique générale (Thouvenot, 1978) où $C$ est le symbole principal caractérisant un système capacitif ou compartimental ef l'indice concerne la localisation anatomique $(D=$ duodénum) ; les chiffres indiquent l'ordre dans le sens du transit.

Le diagramme montre que la distension du premier compartiment duodénal $C D_{1}$ est plus importante que pour le deuxième compartiment $C D_{2}$ dans les mêmes conditions expérimentales de pression et de temps. Les écarts sont tels que ces différences sont significatives pour les pressions imposées $>400 \mathrm{~Pa}$ (test de Student : $\mathrm{P}=0,05$ pour $400 \mathrm{~Pa}$ ef $\mathrm{P}<0,01$ pour les pressions supérieures). Le calcul de la 
compliance $\Delta \mathrm{V} / \Delta \mathrm{P}$ pour chaque pression, montre (tabl. 2) que le comportement élastique des deux compartiments est différent.

TABLEAU 2

Volumes moyens exprimés en $\mathrm{ml}$ avec leur dispersion $\sigma$ pour chaque palier de pression exprimée en $\mathrm{Pa}$ pour les deux compartiments duodénaux étudiés

\begin{tabular}{|c|c|c|c|c|c|c|c|c|c|c|c|c|}
\hline & \multicolumn{4}{|c|}{$\mathrm{CD}_{1} \quad \mathrm{n}=8$} & & & \multicolumn{6}{|c|}{$C D_{2}$} \\
\hline & \multicolumn{4}{|c|}{ Pression en pascals } & & & \multicolumn{6}{|c|}{ Pression en pascals } \\
\hline & 200 & 400 & 600 & 800 & 1000 & 1200 & 200 & 400 & 600 & 800 & 1000 & 1200 \\
\hline $\bar{v} \mathrm{ml} \ldots$. & 0,048 & $0,149 *$ & $0,27 *$ & $0,39 *$ & $0,47 *$ & $0,48 *$ & 0,02 & 0,046 & 0,07 & 0,10 & 0,18 & 0,28 \\
\hline$\sigma \ldots \ldots$ & 0,08 & 0,19 & 0,17 & 0,19 & 0,19 & 0,10 & 0,027 & 0,049 & 0,059 & 0,08 & 0,08 & 0,07 \\
\hline $\begin{array}{l}\text { Comp. } \\
\mathrm{ml} / \mathrm{hPa} \quad \ldots\end{array}$ & 0,024 & 0,037 & 0,045 & 0,049 & 0,047 & 0,04 & 0,01 & 0,011 & 0,011 & 0,012 & 0,018 & 0,023 \\
\hline $\begin{array}{l}d \max \ldots . . \\
d \mathrm{pd} . . . \\
d \mathrm{pr} . . .\end{array}$ & \multicolumn{6}{|c|}{$\begin{array}{l}0,56 \pm 0,08 \\
0,33 \pm 0,07 \\
0,44 \pm 0,06\end{array}$} & \multicolumn{6}{|c|}{$\begin{array}{l}0,45 \pm 0,08 \\
0,13 \pm 0,05 \\
0,17 \pm 0,06\end{array}$} \\
\hline$\frac{d p d}{d \max } \cdots$ & \multicolumn{6}{|c|}{58 p. 100} & \multicolumn{6}{|c|}{29 p. 100} \\
\hline$\frac{d p r}{d \max } \cdots$ & \multicolumn{6}{|c|}{79 p. 100} & \multicolumn{6}{|c|}{38 p. 100} \\
\hline
\end{tabular}

Les probabilités $P$ (test $t)$ sont indiquées : compliance et diamètres moyens correspondant au modèle cylindrique pour une longueur de $2 \mathrm{~cm}$.

Diamètre du modèle cylindrique correspondant au seuil de l'activité pendulaire ( $d$ pd) ou propulsive ( $d p r$ ). Les rapports $d p d / d$ max et $d p r / d p$ max indiquent ces seuils en $p .100$ du diamètre maximal ( $d$ max).

* Ces valeurs sont significatives dans l'intervalle $0,01<P<0,05$.

On a précisé également, pour chaque volume intraluminal moyen ef par unité de longueur, le diamètre théorique de l'intestin supposé cylindrique. L'intérêt de ce calcul est de situer les caractéristiques métriques correspondant au déclenchement des activités motrices enregistrées parallèlement. Celles-ci apparaissent de deux types : les rythmes pendulaires avec balancement ou torsion longitudinale sans contraction circulaire apparente ef les contractions qui mettent en jeu les fibres circulaires : segmentation rythmique locale ou propulsion péristaltique propagée. Les contractions pendulaires entraînent des variations de volume faibles mais non négligeables $(0,01$ à $0,02 \mathrm{ml}$ ) et pour le péristaltisme les variations de volume à chaque cycle atteignent 0,02 à $0,05 \mathrm{ml}$.

Le tableau 2, montre que pour $C D_{1}$ le début des contractions pendulaires correspond à un diamètre théorique de $0,33 \pm 0,07 \mathrm{~cm}$ correspondant à $58 \mathrm{p}$. 100 de la dilatation maximale. Pour $\mathrm{CD}_{2}$, le début des contractions correspond à 29 p. 100 de la dilatation maximale, soit un diamètre théorique de : $0,13 \pm 0,05 \mathrm{~cm}$.

Le péristaltisme se déclenche pour une dilatation de 79 p. 100 pour $C D_{1}$ ef de 38 p. 100 pour $C D_{2}$. Ainsi, d'une manière générale, le seuil des activités motrices est plus bas pour le deuxième compartiment que pour le premier. 


\section{Discussion.}

In vivo, l'étude radiologique du transit baryté et plus particulièrement les formes observées, avaient fait pressentir l'existence au niveau du grêle, de compartiments relativement stables et hiérarchisés selon deux fonctions : fonction réservoir pour un segment radiologiquement distendu $\left(C D_{1}\right)$ et fonction de transport pour le segment $\left(C D_{2}\right)$ plus étroit et plus long. Si après prélèvement, on place ces segments dans des conditions comparables, mais in vitro, l'épreuve fonctionnelle que constitue la distension à pressions imposées, nous a révélé des différences significatives dans le comportement de ces deux segments. Pour une même plage de pressions, le premier présente une capacité plus importante et se laisse distendre passivement. De plus, l'activité motrice volumétrique n'apparaît qu'à un stade tardif de la distension (> 58 p. 100 de la dilatation maximale). Le second compartiment apparaît moins distensible et de plus son activité motrice débute plus précocement ( 28 p. 100 de la dilation maximale).

Ces résultats significatifs permettent de confirmer l'existence au niveau du duodénum d'un premier compartiment à fonction capacitive prédominante (succédant à l'antre pylorique) tandis que la portion suivante (débutant avec le sphincter d'Oddi) possède des caractéristiques biomécaniques qui le font classer dans le cadre d'un. système de transport. Quant aux activités pendulaires réputées pour n'effectuer aucun pompage et ne mettant en jeu aucune activité des fibres circulaires, elles apparaissenł ici, susciter des variations de volume non négligeables.

Il faut préciser également pour l'étape in vivo, que les caractéristiques duodénales ainsi définies correspondent à un état pseudostationnaire dans la période post-prandiale (1-10 min après ingestion). On doit se réserver la possibilité de caractériser d'autres états plus tardifs pour lesquels la structuration compartimentale et ses caractéristiques sont différentes.

Enfin, pour une même expérience de distension in vitro, correspondant à un état donné in vivo, chaque palier de distension dans sa chronologie imposée peut modifier le métabolisme, l'excitabilité ef la contractilité du muscle lisse. Ce type de comportement élastique entrant dans le cadre des structures dépendantes des états antérieurs ou structures diachroniques (Fung, 1972).

Journées Ingestion-Digestion-Absorption de l'Association française de Nutrition, Paris, 15-16 novembre 1979.

\section{Références}

FUNG Y. C., 1972. Stress-strain-history relations of soft tissues in simple elongation. In Biomechanics. Its foundations and objectives. Prentice-Hall Inc. Englewood Cliffs New-Jersey, 641 pp.

MORELLE O., GAUDEAU C., THOUVENOT J., 1979. Cinétique quantitative comparée d'une suspension barytée avec ou sans glucides chez le rat éveillé. Ann. Biol. anim. Bioch. Biophys., 19, 911-914.

MORELLE O., 1978. Sur la conception compartimentale du transit digestif : $1^{\circ}$ Méthodologie intégrant le traitement d'images radiologiques. $2^{\circ}$ Approche du rôle des stimuli volumétriques ef biochimiques. Th. Biol. Hum., Fac. Méd., Tours, 154 pP.

RIVAILLIER J., LONGEVIALLE C., GAUDEAU C., THOUVENOT J., 1978. Machine d'acquisition et de traitement d'images en mode conversationnel. C. R. Journ. d'Informatique Médicale, Toulouse, I. R. I. E. S., 13-17 mars, 1, 295-310.

THOUVENOT J., 1978. Méthode de simulation symbolique des systèmes physiologiques pour l'enseignement et la recherche. J. Physiol., 74, 19A. 